\title{
The Decreasing Incidence of Acute Appendicitis During COVID-19: A Retrospective Multi-centre Study
}

\author{
James Tankel $^{1} \cdot$ Aner Keinan $^{1} \cdot$ Ori Blich ${ }^{1} \cdot$ Michael Koussa $^{1,2} \cdot$ Brigitte Helou $^{3}$ • \\ Shahaf Shay ${ }^{4} \cdot$ Diaa Zugayar $^{3}$ - Alon Pikarsky ${ }^{3} \cdot$ Haggi Mazeh $^{4} \cdot$ Ram Spira $^{1}$. \\ Petachia Reissman ${ }^{1}$
}

Published online: 26 May 2020

(C) Société Internationale de Chirurgie 2020

\begin{abstract}
Background As the novel coronavirus disease 19 (COVID-19) spreads, a decrease in the number of patients with acute appendicitis (AA) has been noted in our institutions. The aim of this study was to compare the incidence and severity of AA before and during the COVID-19 pandemic.

Methods A retrospective cohort analysis was performed between December 2019 and April 2020 in the four highvolume centres that provide health care to the municipality of Jerusalem, Israel. Two groups were created. Group A consisted of patients who presented in the 7 weeks prior to COVID-19 first being diagnosed, whilst those in the 7 weeks after were allocated to Group B. A comparison was performed between the clinicopathological features of the patients in each group as was the changing incidence of AA.

Results A total of 378 patients were identified, 237 in Group A and 141 in Group B (62.7\% vs. 37.3\%). Following the onset of COVID-19, the weekly incidence of AA decreased by $40.7 \%(p=0.02)$. There was no significant difference between the groups in terms of the length of preoperative symptoms or surgery, need for postoperative peritoneal drainage or the distribution of complicated versus uncomplicated appendicitis.

Conclusions The significant decrease in the number of patients admitted with AA during the onset of COVID-19 possibly represents successful resolution of mild appendicitis treated symptomatically by patients at home. Further research is needed to corroborate this assumption and identify those patients who may benefit from this treatment pathway.
\end{abstract}

James Tankel

jamietankel@hotmail.com

1 Department of General Surgery, Shaare Zedek Medical Centre, The Hebrew University School of Medicine, Jerusalem, Israel

2 Department of General Surgery, St Joseph Hospital, Jerusalem, Israel

3 Department of Surgery, Hadassah-Hebrew University Medical Centre, Ein Kerem Campus, Jerusalem, Israel

4 Department of Surgery, Hadassah-Hebrew University Medical Centre, Mount Scopus Campus, Jerusalem, Israel

\section{Introduction}

Since first being detected in December 2019, the novel severe acute respiratory syndrome coronavirus 2 (SARS$\mathrm{CoV}-2$ ) has spread worldwide and has been classified as a global pandemic of international concern. Up to $15 \%$ of those affected with the acute form of the illness, termed coronavirus disease 19 (COVID-19), present with a severe phenotype of the disease. The resulting impact on health resources worldwide has been innumerable [1]. As COVID-19 is still spreading, the exact impact that this virus will have leaves much to be discovered. Whilst a majority of the published literature regarding COVID-19 has explored disease transmission characteristics, 
pathogenesis, treatment options and patient outcomes, the impact on other areas of medicine, including general surgery, is yet to be investigated.

Acute appendicitis (AA) is the most common emergency faced by the general surgeon representing $4.5 \%$ of all cases of abdominal pain [2]. Over 250,000 appendectomies are performed each year in the USA alone [3]. Whilst surgery is still considered the gold standard for the treatment of AA, a conservative approach has been advocated in selected cases using antibiotics as a first-line treatment. Nevertheless, cases of suspected appendicitis are often referred to the Department of Emergency Medicine (ED) for surgical evaluation and are not treated by primary care physicians. In comparison, other causes of an acute abdomen, such as mild biliary complaints and recurrent diverticulitis, may be managed successfully in the primary care setting without surgical review in the ED [4, 5]. Thus, the incidence of AA diagnosed in the ED likely reflects the overall incidence AA in the general population.

It has been noted that during outbreaks of influenza and Middle East Respiratory Syndrome (MERS) [6, 7], ED referrals for non-respiratory cause decreased significantly as the epidemics progressed. An outbreak of respiratory disease should not by itself affect the incidence of AA. Nevertheless, we have noticed a significant decline in the number of cases of AA presenting to our institutions, whilst the number of COVID-19 cases increases. Thus, the aim of this study was to explore the impact of COVID-19 on the number and characteristics of patients presenting to the ED with AA at all of the hospitals in the municipality of Jerusalem, Israel.

\section{Materials and methods}

This multi-centre retrospective cohort analysis was undertaken at four high-volume university-affiliated teaching hospitals that provide health care to the entire Jerusalem metropolitan and surrounding area. Collectively, they have a catchment area of approximately 1.3 million people.

Approval was sought from the ethics committee at each hospital. The requirement for informed consent was waived by each centre in the light of the retrospective and anonymous nature of the study. Using Israeli Ministry of Health diagnostic codes 47-47.19 based on the ICD-9 codes for appendicitis, all consecutive patients between 31 December 2019 and 07 April 2020 were identified via a search of each hospital's electronic databases which are kept in real time. The incidence of confirmed COVID-19 infections was extrapolated from the Israeli Ministry of Health figures which are published daily on their website.

Cases identified were verified according to the following inclusion criteria: Patients had to have presented emergently via the ED; have either a radiologically or intra-operatively diagnosed AA; and have electronic medical records available. With regard to treatment, patients were included if they were treated surgically with laparoscopic or open appendectomy or conservatively with intravenous antibiotics. Delayed presentation, defined as attendance to the ED more than 3 days after the onset of symptoms, did not prevent inclusion in the study. Patients presenting with complications of a delayed presentation, such as phlegmonous or perforated appendicitis, or with a peri-appendicular abscess were identified using the same diagnostic codes and included in the analysis as well. Those patients who underwent surgery for a presumed AA and who were subsequently found to have a grossly normal appendix were also included in the study. Finally, both children and adults were also included.

Data extracted from patients notes included demographic and clinicopathological variables and the postoperative length of stay. Intra-operative findings were recorded and classified as AA if gross acute inflammation of the appendix was seen. Complicated AA referred to the presence of gangrene, perforation or a peri-appendicular abscess. Finally, a normal appendix was defined as the absence of gross inflammatory changes. Serious postoperative complications were also recorded if they occurred within 30 days following surgery defined as being of Clavien-Dindo grade $\geq 3$.

The primary outcome of the study was to compare the incidence of AA before and during the onset of COVID-19. The secondary outcome was to understand, whether as a result of this potential change in the incidence of $\mathrm{AA}$, there was a change in the characteristics or severity of patients diagnosed with AA during the COVID-19 period compared to beforehand.

In order to assess these outcomes, the cohort was split into two groups. Those patients presenting in the 7 weeks before the first case of COVID-19 was diagnosed in Israel were allocated to Group A, whilst those presenting in the 7 weeks after were allocated to Group B. This corresponded to the 31 December 2019-18 February 2020 for the former and 19 February 2020-07 April 2020 for the latter.

In order to evaluate whether the change in the weekly incidence of AA was either temporal or seasonal in nature rather than due to COVID-19, the weekly incidence of AA during the equivalent period 12 months prior to the dates allocated for Group B was also calculated.

All statistical analysis was performed using SPSS, version 21 (IBM SPSS Statistics for Windows, version 21.0. Released 2012. Armonk, NY: IBM Corp). Descriptive statistics are displayed as mean or $\mathrm{N}$ with standard deviation or percentage in parenthesis unless stated otherwise. Univariate comparisons between the two groups were 
performed using Chi-squared or Fisher's exact test for ordinal or categorical variables. For continuous variables, either the Mann-Whitney U test or the Student's T test was used as appropriate. A $p$ value of $<0.05$ was considered significant.

The incidence of AA was calculated weekly with the change in the number of weekly cases compared between each 7-week period. This was plotted against the national increase in new diagnoses of COVID-19.

\section{Results}

Between the study dates, 378 patients with AA were collectively admitted via the ED of all of the hospitals included with 237 in Group A and 141 in Group B $(62.7 \%$ vs $37.3 \%$ ). The basic demographic and clinical data for these patients are described in Table 1 as is a comparison between Groups A and B.

When comparing between the groups, there was no significant difference in the distribution of age or sex of the patients, the length of symptoms prior to ED attendance nor the number of patients who presented with a neutrophilia. The difference in the distribution of the severity of appendicitis also failed to reach a level of statistical significance. Similarly, the number of patients with a delayed presentation or for whom postoperative peritoneal drainage was required did not significantly vary between the two groups.
A subset analysis of the severity of AA was also performed between Groups A and B. For the purpose of this analysis, rather than comparing the group as a whole the first week from Group A was compared with the first week from Group B and so on. There was no significant difference found in the distribution of the severity of AA between the two groups irrespective of the individual week explored.

In order to analyse the change in the incidence of AA between Groups A and B the rate of change, expressed as the average weekly change in the number of cases of AA over the entire 7-week period, was calculated. The results of this analysis are described in Table 2. There was a significant difference in the distribution of cases between the two groups $(p=0.020)$ with the rate of change smaller for Group A (-1.9) than for Group B (- 4.5) representative of a $40.7 \%$ decrease in the number of cases over the 7-week period for the latter. This relationship is also displayed in Fig. 1 demonstrating the weekly number of cases of AA for each group plotted against the weekly incidence of new national COVID-19 diagnoses.

Figure 2 displays the weekly incidence of AA for Group $\mathrm{B}$ compared with the incidence from the equivalent period 12 months prior. Whilst for the former the incidence of AA decreased, for the later an opposite trend was noted. The distribution of the weekly incidence of AA differed significantly $(p=<0.001)$.

Table 1 A comparison between Groups A and B of the demographic and clinical data of patients presenting with AA

\begin{tabular}{|c|c|c|c|c|}
\hline Variable & $\begin{array}{l}\text { Total } \\
N=378\end{array}$ & $\begin{array}{l}\text { Group A } \\
N=237\end{array}$ & $\begin{array}{l}\text { Group B } \\
N=141\end{array}$ & $p$ value \\
\hline Age (years) & $23.2( \pm 16.7)$ & $23.1( \pm 16.7)$ & $23.3( \pm 16.8)$ & 0.841 \\
\hline \multicolumn{5}{|l|}{ Sex } \\
\hline Male & $217(57.4 \%)$ & $140(59.1 \%)$ & $77(54.6 \%)$ & \multirow[t]{2}{*}{0.396} \\
\hline Female & $161(42.6 \%)$ & $97(40.9 \%)$ & $64(45.4 \%)$ & \\
\hline Neutrophilia on presentation $\left(>11 \times 10^{9 / \mathrm{L}}\right)$ & $257(68.0 \%)$ & $154(65.0 \%)$ & $103(73.0 \%)$ & 0.103 \\
\hline Length of symptoms (days) & $1.7( \pm 1.9)$ & $1.8( \pm 2.3)$ & $1.5( \pm 0.84)$ & 0.726 \\
\hline Delayed presentation ( $>3$ days) & $18(4.8 \%)$ & $14(5.9 \%)$ & $4(2.8 \%)$ & 0.150 \\
\hline \multicolumn{5}{|l|}{ Treatment modality } \\
\hline Conservative & $46(12.2 \%)$ & $35(14.8 \%)$ & $11(7.8 \%)$ & \multirow[t]{3}{*}{0.052} \\
\hline Open appendectomy & $10(2.6 \%)$ & $4(1.7 \%)$ & $6(4.3 \%)$ & \\
\hline Laparoscopic appendectomy & $322(85.2 \%)$ & $198(83.5 \%)$ & $124(87.9 \%)$ & \\
\hline Length of surgery (minutes) & $43.3( \pm 26.6)$ & $40.9( \pm 24.8)$ & $47.2( \pm 28.9)$ & 0.726 \\
\hline \multicolumn{5}{|l|}{ Grade of appendicitis } \\
\hline Normal appendix & $3(0.8 \%)$ & $2(0.8 \%)$ & $1(0.7 \%)$ & \multirow[t]{3}{*}{0.167} \\
\hline Uncomplicated appendicitis & $315(83.3 \%)$ & $204(86.1 \%)$ & $111(78.7 \%)$ & \\
\hline Complicated appendicitis & $60(15.9 \%)$ & $31(13.1 \%)$ & $29(20.6 \%)$ & \\
\hline Postoperative peritoneal drain & $29(7.7 \%)$ & $17(7.2 \%)$ & $12(8.5 \%)$ & 0.431 \\
\hline Length of admission (days) & $2.4 \pm 2.3$ & $2.5 \pm 2.4$ & $2.3 \pm 2.2$ & 0.139 \\
\hline Serious complication & $2(0.5 \%)$ & $1(0.4 \%)$ & $1(0.7 \%)$ & $>0.999$ \\
\hline
\end{tabular}


Table 2 The difference in the rate of change of cases of AA between Groups A and B

\begin{tabular}{lllll}
\hline & Number of cases of AA & Average number of cases per week & Rate of change (cases of AA/week) & $p$ value \\
\hline Group A & 237 & 33.9 & -1.9 \\
Group B & 141 & 20.1 & -4.5 \\
\hline
\end{tabular}

Fig. 1 Weekly incidence of AA cases between Groups A and B against the increase in weekly COVID-19 cases. Descriptive caption: whilst a slight decrease in the weekly number of AA is noted in Group A, a marked decrease from 31 to 4 cases per week is noted in Group B. This change occurs with a concurrent increase in the number of COVID-19 cases

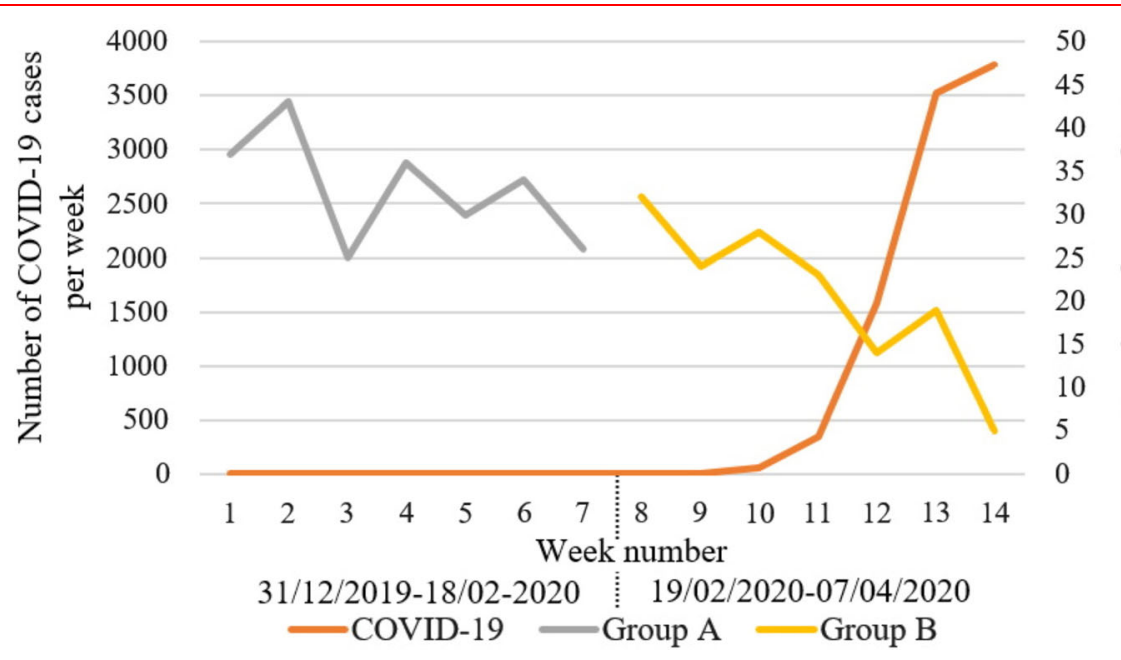

0

35

30

25

20

15

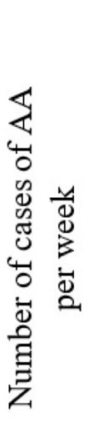

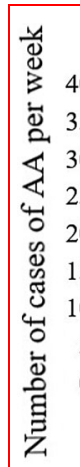

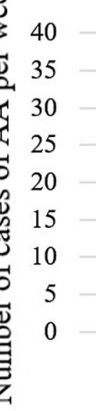

Weekly incidence of AA 19/02/2020-07/04/2020 (Group B)

Weekly incidence of AA 19/02/2019-07/04/2019

Fig. 2 A comparison of the weekly incidence of AA between Group B and the equivalent period one year prior. Descriptive caption: the weekly incidence of AA was similar between Group B and the historical data collected from the equivalent period one year prior. However, after 4 weeks, the incidence of AA for the former increases, whilst for the latter it decreases

\section{Discussion}

In this study, we have shown that the rate of patients presenting to the ED with AA has decreased dramatically during the time that the number of COVID-19 cases diagnosed increased. We have also shown that patients are not presenting to hospital later, do not have an increased incidence of complicated appendicitis, do not have an increased length of stay following surgery and are no more likely to require postoperative peritoneal drainage. The implication is that at a time when global healthcare resource allocation is being scrutinised, with the decrease in the incidence of AA, resources allocation could be diverted away from acute surgical services at times of such desperate need.

There are several possibilities that could explain, at least in part, this trend. It is possible that the decrease in the number of patients presenting to the ED is due to a genuine decrease in the incidence of AA. Indeed, seasonal variations in the incidence of AA have been noted with an increase in the number of cases found during summer months and fewer during the winter [8]. Additionally, the global number of patients suffering from AA seems to be decreasing in recent years [9]. Nevertheless, these reasons fail to explain the rapid decrease in the number of patients presenting with AA in the short period of time noted above. Moreover, in comparison with the decrease in the weekly incidence of AA found during the dates allocated for Group B, during the equivalent dates one year prior the weekly incidence of AA increased. This trend was noted to be statistically significant. This excludes seasonal or temporal changes as an explanation of the decrease in the incidence of AA identified during the COVID-19 period. However, as noted in Fig. 2, it still took approximately 
4 weeks after the first diagnosis of COVID-19 in Israel until a difference was noted in the incidence of AA presenting the ED.

The dates delineating Groups A and B were chosen as the first case of COVID-19 was diagnosed in Israel on the 21 February 2019. Although the changes in patterns of patient referral to the ED took time to mature, it was postulated by the research team that this was because it also took time for the public interest that the epidemic engendered to grow. Nevertheless, when considering the incidence of AA reported for Group A as a whole, it is comparable to reports from other developed countries, whilst that for Group B is far less than would be expected [9].

Outside of the setting of case reports describing H1N1 influenza presenting as AA, the impact of a global pandemic or a local epidemic on the incidence of the acute abdomen has not been reported in the academic literature [10]. The data that do exist have shown that although influenza or MERS outbreaks lead to an increase in respiratory-related presentations to the ED, non-respiratory presentations decline significantly albeit small in absolute terms $[6,7]$.

Considering these changes, rather than a genuine decrease in the incidence of AA, it is possible that patients choose not to present to the ED. The decision may be motivated by several factors. Firstly, the aggressive national policy of social isolation and reduction in public transport that was promoted early in Israel may have contributed significantly to patient's ability to access healthcare services. There may also be an element of patient anxiety and concern about patient-to-patient transmission in the ED as hospitals have been found to be highrisk environments for the transmission of respiratory illnesses during epidemics [11-13].

Patients who present to hospital more than $24 \mathrm{~h}$ following the onset of symptoms are at increased risk of suffering from perforated appendicitis $[14,15]$. Therefore, it would have been expected that if fewer patients were presenting to the $\mathrm{ED}$, a greater incidence of delayed presentations or complicated appendicitis would have been found. However, this was not demonstrated in the cohort presented here.

Treating AA with intravenous antibiotics rather than surgery has been well described in the literature [16-18]. We postulate that those patients who did not present to the ED did not receive antibiotic therapy. This is because right lower quadrant pain found on examination in primary care results in prompt referral for surgical evaluation in the ED in nearly all cases.

In a study by Park et al., the rate of treatment failure in patients with a radiologically confirmed AA was similar among those patients receiving supportive care and those receiving antibiotics [19]. The selection criteria for treating AA without antibiotics in this study were dilatation of the appendix up to a maximum diameter of $11 \mathrm{~mm}$ and the absence of fecaliths. It may well be that the decrease in the number of patients presenting with AA to the ED reflects an increase in the number of patients successfully treating themselves symptomatically at home. This builds previous evidence that describes spontaneous resolution of an acute appendicitis in the absence of complications [20, 21].

There are several limitations to this study. With the evolving nature and growing understanding of the pathogenesis and healthcare impact of COVID-19, the evidence base surrounding the conclusions made here may well change in the future. In the light of the strict restrictions on travel implemented early in the COVID-19 pandemic, generalising the results of the changing nature of ED presentations may not be possible to other cities worldwide. Also, by utilising hospital codes in an effort to identify all patients diagnosed with AA, some patients may have been excluded by this search technique. Whilst it is likely that if a clinical suspicion of AA exists patients would be referred from primary care for surgical review, the study is also limited by an inability to capture those patients who were treated in primary care. As noted above, it took approximately 4 weeks after the onset of COVID-19 for the change in the incidence of AA to become apparent. Despite the week-by-week subset analysis noted above not finding any differences in distribution of the severity of AA, ultimately changing the dates that defined Groups A and B may affect the results of the analyses described above. Finally, some of the patients included in the study had a short follow-up interval and hence late presentations of complications following supportive therapy may not have been accurately recorded.

In conclusion, COVID-19 is a global pandemic challenging healthcare systems worldwide. The significant decrease in the number of patients admitted with AA during the onset of COVID-19 possibly represents successful resolution of mild appendicitis treated symptomatically by patients at home. Further research is needed to corroborate this assumption and identify those patients who may benefit from this treatment pathway, especially in challenging times when healthcare resources are so desperately strained.

Acknowledgements There was no grant support made available for this research.

\section{Compliance with ethical standards}

Conflict of interest All authors declare that they have no conflict of interest. 
Ethical standards This study was approved by the local ethics committee of each hospital who waived the requirement for informed consent.

\section{References}

1. Wu Z, McGoogan JM (2020) Characteristics of and important lessons from the coronavirus disease 2019 (COVID-19) outbreak in China. JAMA, 24 [Epub ahead of print]

2. Cervellin G, Mora R, Ticinesi A, Meschi T, Comelli I, Catena F et al (2016) Epidemiology and outcomes of acute abdominal pain in a large urban Emergency Department: Retrospective analysis of 5,340 cases. Ann Transl Med 4(19):362

3. Addiss DG, Shaffer N, Fowler BS, Tauxe RV (1990) The epidemiology of appendicitis and appendectomy in the United States. Am J Epidemiol 132(5):910-925

4. Johnston MJ, Fitzgerald JEF, Bhangu A, Greaves NS, Prew CL, Fraser I (2014) Outpatient management of biliary colic: a prospective observational study of prescribing habits and analgesia effectiveness. Int J Surg 12(2):169-176

5. Humes DJ (2012) Diagnosing and managing acute diverticulitis. Practitioner 256(1753):21-22

6. Schanzer DL, Schwartz B (2013) Impact of seasonal and pandemic influenza on emergency department visits, 2003-2010, Ontario. Canada Acad Emerg Med 20(4):388-397

7. Paek SH, Kim DK, Lee JH, Kwak YH (2017) The impact of middle east respiratory syndrome outbreak on trends in emergency department utilization patterns. J Korean Med Sci 32(10):1576-1580

8. Stein GY, Rath-Wolfson L, Zeidman A, Atar E, Marcus O, Joubran $S$ et al (2012) Sex differences in the epidemiology, seasonal variation, and trends in the management of patients with acute appendicitis. Langenbeck's Arch Surg 397(7):1087-1092

9. Ferris M, Quan S, Kaplan BS, Molodecky N, Ball CG, Chernoff GW et al (2017) The global incidence of appendicitis: a systematic review of population-based studies. Ann Surg 266(2):237-241

10. Cunha BA, Pherez FM, Durie N (2010) Swine influenza (H1N1) and acute appendicitis. Hear Lung $\mathrm{J}$ Acute Crit Care 29(6):544-546

11. Alenazi TH, Al Arbash H, El-Saed A, Alshamrani MM, BaffoeBonnie H, Arabi YM et al (2017) Identified transmission dynamics of middle east respiratory syndrome coronavirus infection during an outbreak: implications of an overcrowded emergency department. Clin Infect Dis 65(4):675-679

12. Hastings DL, Tokars JI, Abdel Aziz IZAM, Alkhaldi KZ, Bensadek AT, Alraddadi BM (2016) Outbreak of middle east respiratory syndrome at tertiary care hospital, Jeddah, Saudi Arabia, 2014. Emerg Infect Dis 22(5):794-801

13. Assiri A, McGeer A, Perl TM, Price CS, Al Rabeeah AA, Cummings DAT et al (2013) Hospital outbreak of middle east respiratory syndrome coronavirus. N Engl J Med 369:407-416

14. Williams N, Bello M (1998) Perforation rate relates to delayed presentation in childhood acute appendicitis. J R Coll Surg Edinb 43(2):101-102

15. Kearney D, Cahill RA, O'Brien E, Kirwan WO, Redmond HP (2008) Influence of delays on perforation risk in adults with acute appendicitis. Dis Colon Rectum 51(12):1823-1827

16. Di Saverio S, Sibilio A, Giorgini E, Biscardi A, Villani S, Coccolini F et al (2014) The NOTA study (non operative treatment for acute appendicitis): Prospective study on the efficacy and safety of antibiotics (amoxicillin and clavulanic acid) for treating patients with right lower quadrant abdominal pain and long-term follow-up of conservatively treated suspected appendicitis. Ann Surg 260(1):109-117

17. Armstrong J, Merritt N, Jones S, Scott L, Bütter A (2014) Nonoperative management of early, acute appendicitis in children: Is it safe and effective? J Pediatr Surg 49(5):782-785

18. Georgiou R, Eaton S, Stanton MP, Pierro A, Hall NJ (2017) Efficacy and safety of nonoperative treatment for acute appendicitis: a meta-analysis. Pediatrics 39(3):e20163003

19. Park HC, Kim MJ, Lee BH (2017) Randomized clinical trial of antibiotic therapy for uncomplicated appendicitis. Br J Surg 104(13):1785-1790

20. Kirshenbaum M, Mishra V, Kuo D, Kaplan G (2003) Resolving appendicitis: role of CT. Abdom Imaging 28(2):276-279

21. Andersson RE (2007) The natural history and traditional management of appendicitis revisited: spontaneous resolution and predominance of prehospital perforations imply that a correct diagnosis is more important than an early diagnosis. World J Surg 31(1):86-92. doi:https://doi.org/10.1007/s00268-006-0056-y

Publisher's Note Springer Nature remains neutral with regard to jurisdictional claims in published maps and institutional affiliations. 